\title{
Molecular Genetic Testing for Kidney Disorders During the COVID-19 Pandemic
}

Susan M. Kirwin; ${ }^{1}$ Katherine M. Robbins, Ph.D. $;{ }^{2}$ Kathleen M.B. Vinette $;{ }^{3}$ Lee Hirata $;{ }^{4}$ Karen W. Gripp, M.D. ${ }^{5}$ Vicky L. Funanage, Ph.D. ${ }^{6}$

1. Nemours Children's Health, Molecular Diagnostics Laboratory

2. Nemours Children's Health, Molecular Diagnostics Laboratory

3. Nemours Children's Health, Molecular Diagnostics Laboratory

4. Nemours Children's Health, Molecular Diagnostics Laboratory

5. Nemours Children's Health, Molecular Diagnostics Laboratory

6. Nemours Children's Health, Molecular Diagnostics Laboratory

\begin{abstract}
Chronic kidney disease (CKD) has major morbidity and mortality for children and adults. While in adults $\mathrm{CKD}$ often is associated with diabetic complications, genetic variants can be the underlying cause in both populations. Beginning in 2016 with the emergence of more affordable next-generation sequencing (NGS) technologies, the Molecular Diagnostics Lab at Nemours Children's Hospital-Delaware developed the first clinically actionable pediatric NGS kidney panel comprised of 46 genes including APOL1. Apolipoprotein L1 (APOL1) associated nephropathy is reported along a spectrum of non-diabetic kidney disease. It is significantly associated with two "risk alleles" defined as G1 and G2 and typically found in individuals of African descent. In early 2020, as COVID-19 spread across the globe, reports of patients with kidney failure began to emerge. A collapsing glomerulopathy in Black patients with COVID-19 was found to be associated with the $A P O L 1$ predisposition of the known G1 and/or G2 risk variants. We identified genetic variants in 11 genes (NPHS1; NPHS2; LAMB2; WT1; COL4A4; COL4A5; COQ8B; CUBN; MEFV; PMM2; SMARCAL1) known to be associated with pediatric onset nephrotic syndrome, or detection of the high-risk haplotype of $A P O L 1$, in the majority (78\%) of patients tested. These clinically actionable results guided medical care and improved patient outcomes.
\end{abstract}

\section{Introduction}

Chronic kidney disease (CKD) has major morbidity and mortality for children and adults. While in adults it often is associated with diabetic complications, genetic variants can be the underlying cause in children and adults alike. With a prevalence of 1.5 - 3 per million, many of the children under age 16 years diagnosed annually with CKD have an underlying genetic cause or predisposition. ${ }^{1-3}$ While linked to morbidity and mortality, CKD is also associated with an increase in cardiovascular disease. Focal segmental glomerulosclerosis (FSGS) is a pathologic finding in many renal disorders presenting with proteinuria and declining renal function. Nephrotic syndrome is typically defined by edema, hypoalbuminemia, hyperlipidemia, and proteinuria, and can lead to FSGS and end-stage renal disease (ESRD). These patients can be classified by their response to steroid treatment: steroid sensitive nephrotic syndrome (SSNS) or steroid resistant nephrotic syndrome (SRNS). In children and young adults, approximately 80\% 
respond to steroid treatments. The remaining SRNS population can progress to CKD and ESRD. Next-generation sequencing (NGS) based sequencing for genetic variants involved in SRNS or FSGS can inform diagnosis and guide the healthcare provider in treatment.

Collapsing glomerulopathy (CG) is a subtype of FSGS and reported to be the most common in individuals of African descent. ${ }^{4}$ Although CG is not common in young children, $30 \%$ of pediatric patients with FSGS have an underlying genetic cause identifiable through next generation sequencing.

Monogenic forms of FSGS are associated with variants in genes expressed in either the podocyte or the glomerular basement membrane. Pediatric forms are typically categorized as: congenital onset (NPHS1, NPHS2, WT1, LAMB2, PLCE1, LMX1B), infantile onset (ACTN4, COQ2, COQ6), or adolescent onset ( $L A M B 2, C O L 4 A 3, C O L 4 A 4, C O L 4 A 5)$. In addition, several syndromic disorders (Alport syndrome, Denys-Drash syndrome, Alstrom syndrome) often present with kidney disease.

Variants in the gene apolipoprotein L1 (APOL1) are found in individuals of sub-Saharan African ancestry, and these risk variants display a strong association with kidney disease. ${ }^{5}$ The $A P O L 1$ risk haplotypes are defined as G1, comprised of two missense changes (p.Ser358Gly, rs $73885319+$ p.Ile384Met, rs60910145) and a 6 base-pair deletion (p.Asn388_Tyr389del, rs71785313) resulting in a deletion of two amino acids, designated G2. The G1 variants are in almost complete linkage disequilibrium and found on the same chromosome. The high-risk genotypes are designated $\mathrm{G} 1 / \mathrm{G} 1, \mathrm{G} 1 / \mathrm{G} 2$, and $\mathrm{G} 2 / \mathrm{G} 2$ and are considered contributors to the incidence of ESRD in Black children and adults.

Approximately 13\% of Black Americans carry two risk alleles, markedly increasing their risk for a wide range of nondiabetic kidney disease. Yusuf et al report the frequency of G1 in the U.S. at $20-42 \%$, with G2 detected at approximately $15 \%{ }^{6}$ These risk alleles have also been reported in populations from western Africa, African and Hispanic Caribbean, and Brazilian and South American ancestry. ${ }^{6}$ Renal disease in individuals with two APOL1 risk alleles has been described as a "two hit" disease. $A P O L 1$ risk variants represent the "first hit," with the "second hit" being an external trigger such as virus exposure (HIV, CMV, parvovirus B19), medication (interferons, pamidronate, anabolic steroids), or autoimmune disease. ${ }^{4,7,8}$

Throughout the SARS-CoV2 pandemic, reports of individuals with COVID-19 and acute kidney injury (AKI) with high levels of proteinuria, and in some cases collapsing glomerulopathy, emerged from across the globe. Caza et al report 37\% of patients hospitalized with confirmed COVID-19 were diagnosed with AKI. ${ }^{9}$

\section{Methods}

The Molecular Diagnostic Laboratory (MDL) utilized a custom panel designed by the SureSelect Design software (Agilent Technologies, Inc.) to analyze genomic DNA isolated from whole blood (Qiagen). Genomic DNA was sheared using a M220 Ultrasonicator (Covaris, Inc.) prior to library preparation and target enrichment by using the SureSelect XT kit protocol (Agilent Technologies, Inc.). Prepared libraries enriched for the coding exons and exon boundaries of genes associated with steroid resistant nephrotic syndrome and related disorders were sequenced using a MiSeq System (Illumina, Inc.). 
Data analysis was performed using NextGENe Sequence Analysis Software (Version 2.4.2.2, SoftGenetics, LLC.) to align patient data to reference sequences (hg19/GRCh37). Confirmation of all likely pathogenic or pathogenic variants, as well as variants of uncertain significance, was performed using a standard PCR protocol followed by Sanger sequencing (BigDye Terminator v.3.1) and analyzed on a 3500 Genetic Analyzer (ThermoFisher/Applied Biosystems). Sequences were analyzed using MacVector software (MacVector, Inc.). Regions known to have low coverage $(<30 \mathrm{X})$ were also analyzed using standard PCR and Sanger sequencing protocols. All variations from the reference sequence(s) were checked against current literature, the NCBI SNP database, and computational and splice predictions websites. Mutation nomenclature follows guidelines of the Human Genome Variation Society (http://www.hgvs.org).

Single gene testing for $A P O L 1$ G1/G2 was performed by PCR of the last exon of $A P O L 1$, followed by bidirectional sequencing, and compared to the known $A P O L 1$ reference sequence using MacVector software.

\section{Variant Analysis}

Variant classification follows the American College of Medical Genetics (ACMG) Standards and guidelines for the interpretation of sequence variants. All genetic variants possibly associated with phenotype and incidental findings were classified using NextGENe software (SoftGenetics, LLC). Variants classified as pathogenic or likely pathogenic were reported with gene variant assessments. Variants of unknown significance (VUS) were also reported with the information that there was insufficient evidence to support either a likely benign or likely pathogenic classification. APOL1 single gene sequencing was analyzed by alignment to the $A P O L 1$ reference sequence and assessed for the G1 or G2 variant.

\section{Results}

The NGS panel was designed to target 46 genes associated with the rare SRNS disorders. In the small cohort of pediatric patients screened with this FSGS panel over the course of the past two years, we have identified two patients who were initially diagnosed with congenital nephrotic syndrome, at 5 months and 14 months of age respectively. The first patient (unilateral nephrectomy and pathology consistent with mesangial sclerosis) was homozygous for a known pathogenic variant in NPHS1: c.3250dupG, leading to a substitution of glycine for valine and premature stop codon encountered with exon 24 . The second child was first diagnosed around 14 months of age with a two-week history of swelling, edema, nephrotic range proteinuria and hypertension. A renal biopsy was inconclusive. The patient underwent research testing and found to have an autosomal dominant $W T 1$ variant c. $1384 \mathrm{C}>\mathrm{T}$ leading to a substitution of tryptophan for an arginine, and the MDL confirmed this result with our clinical NGS panel.

A rare disorder was identified in a 14-year-old with a homozygous variant cubilin $(C U B N)$ associated with Imerslund-Grasbeck syndrome (Megaloblastic anemia 1). Variants in CUBN cause vitamin B12 malabsorption and proteinuria, and most reported variants are found in the binding domain important for renal protein reabsorption. The identified $C U B N$ variant, a 7 base pair deletion leading to a frameshift and premature stop within exon 45 , was not previously described in a homozygous state, but cited in the literature in compound heterozygosity with another pathogenic variant. The medical management for this patient was changed due to the confirmation of this $C U B N$ variant. 
The NPHS2 gene encodes the slit diaphragm protein podocin, and variants in this gene are among the most common causes of childhood and adolescent nephrotic syndrome. ${ }^{10} \mathrm{~A} 7$-yearold with FSGS was compound heterozygous for a known pathogenic variant within exon 7 of NPHS2, and a second variant with pathogenicity dependent upon the location of the first variant. This exon 5 variant replaces an arginine with a glutamine and is reported with variable frequency in European, South Asian, African, and Latino populations. The pathogenicity of the exon 5 missense variant is reported to be dependent upon a second variant. It is considered pathogenic only when it is located in trans with another variant within exon 7 or 8 . The pathogenic association is reported to cause a less severe phenotype with slowly progressive FSGS.

Alport syndrome (AS), caused by variants in the type IV collagen genes (COL4A3, COL4A4, $C O L 4 A 5)$ can be phenotypically diverse with isolated kidney disease, or progressive disease including extrarenal abnormalities with hearing loss and ocular anomalies. Approximately 85\% of Alport syndrome is X-linked (COL4A5) with autosomal recessive inheritance ( $C O L 4 A 3$, $C O L 4 A 4)$ and rare autosomal dominant cases (COL4A3) comprising the remaining $15 \%$. Two adolescents were found to carry type IV collagen gene variants. The first patient had a history of FSGS (proteinuria, hyperkalemia, and hyponatremia). This same COL4A4 variant had previously been detected in a parent who was reportedly asymptomatic. The second individual diagnosed with Alport syndrome had a history of hematuria, and proteinuria. A variant in the X-linked COL $4 A 5$ affected a highly conserved glycine residue in the triple helical region of the COL $4 A 5$ protein, where pathogenic missense variants frequently occur.

The high-risk APOL1 haplotypes were detected in several pediatric patients tested by our NGS panel. For one individual with FSGS, previous testing at another laboratory did not provide a molecular diagnosis as APOL1 was not included on the panel. Additional testing at the MDL confirmed a homozygous G2/G2 genotype, therefore explaining the patient's phenotype.

Overall, $78 \%$ of the patients tested had a genetic variant which explained their clinical phenotype with the $A P O L 1$ and $C U B N$ genes representing the highest proportion of variants. As shown in figure 1, eighteen patients were screened for genetic variants in the NGS panel. Fourteen patients were found to carry at least one relevant genetic variant, and the frequency distribution of the affected genes is shown. Pathogenic or likely pathogenic variants were associated with the following genes: $A P O L 1$, apolipoprotein 1; COL4A4/COL4A5 type IV collagens alpha 4 and 5; $C O Q 8 B$, co-enzyme Q 8B; $C U B N$, cubilin; $L A M B 2$, laminin beta-2; $M E F V$, familial Mediterranean fever; NPHS1, nephrin; NPHS2, podocin; PMM2, phosphomannomutase-2; SMARCAL1, SWI/SNF related, matrix associated, actin dependent regulator of chromatin, subfamily a like $1 ; W T 1$, Wilms tumor suppressor 1.

Figure 1. Results of next-generation sequencing (NGS) testing in patients referred for FSGS genetic evaluation. 
Doi: $10.32481 /$ djph.2021.12.008

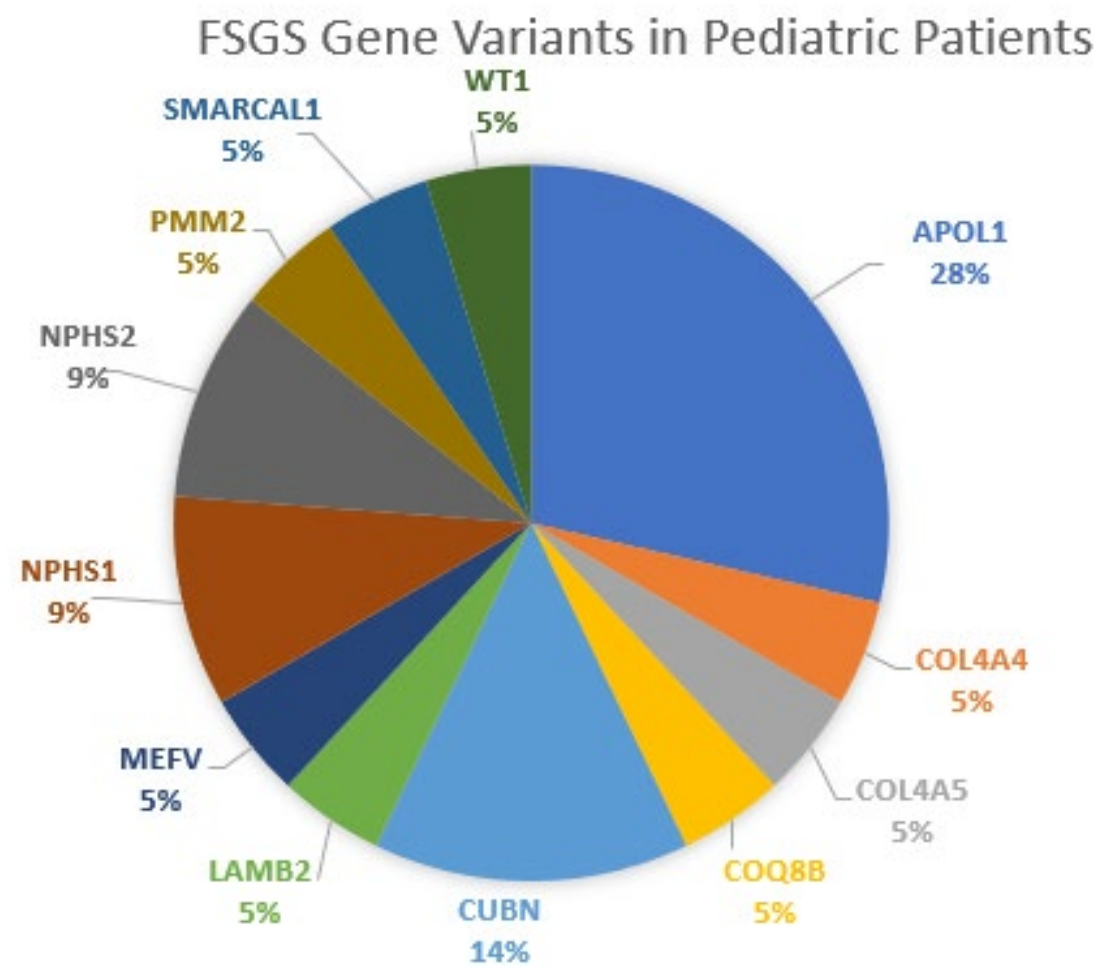

Genotype testing for $A P O L 1$ risk alleles is orderable as either a single gene test to confirm risk allele status, or as part of the 46 gene NGS kidney disease panel. Over the course of the pandemic, we have seen an increased interest in APOL1 testing. As new information emerged that collapsing glomerulopathy was associated in Black patients presenting with COVID-19 and renal issues, the $A P O L 1$ gene was likely considered more frequently in clinical requests for diagnostic testing.

In samples received in the MDL from March 2020 - October 2021 for APOL1 genotyping, 30\% were positive for two risk alleles, and an additional 39\% carried one risk allele. These numbers align with the overall $A P O L 1$ testing profile for individuals with one or two risk alleles in all samples tested in the MDL since 2014. This pre-disposition to renal disease has been highlighted during the pandemic, as Black Americans are at higher risk due to their genetic susceptibility associated with the $A P O L 1$ high-risk genotype.

\section{Discussion}

We identified genetic variants in 11 genes (NPHS1, NPHS2, LAMB2, WT1, COL4A4, COL4A5, $C O Q 8 B, C U B N, M E F V, P M M 2$, SMARCAL1) associated with pediatric onset nephrotic syndrome, as well as detection of the high-risk haplotype of $A P O L 1$. While NGS panels are an appropriate method for the molecular evaluation of nephrotic syndrome, single gene testing for some disorders (such as APOL1-related nephropathy) remain a relevant screening tool. Clinical testing has previously shown that using NGS for detection of multiple monogenic diseases adds to the diagnostic yield, and can lower the turnaround time for molecular diagnoses, thereby improving care. Differentiating FSGS into SSNS and SRNS can improve clinical management 
and outcomes, by decreasing exposure to ineffective treatments and prompt use of more appropriate therapies.

In a 2021 consensus statement on APOL1, Freedman et al. determined that APOL1-associated nephropathy includes a range of kidney diseases, which may be present in children as FSGS, or may appear later in life (Hypertensive nephrosclerosis, NDCKD), or after exposure to a "second hit" (autoimmune disease, HIV, another comorbidity). ${ }^{11}$ Although most individuals with two risk alleles will not develop renal disease, much is unknown regarding the involvement of viral exposure such as SARS-CoV2. ${ }^{12}$ COVID-19 associated glomerulopathy is discussed in 37 peerreviewed articles in PubMed (https://pubmed.ncbi.nlm.nih.gov/?term=COVID-19+and+APOL1), and more than 500 articles mention COVID-19 and AKI. While most people experience mild or moderate COVID-19, approximately 5\% suffer severe symptoms, including respiratory distress, fatigue, muscle and joint pain, headache, and loss of taste or smell. Other affected organ systems include heart, brain, and kidneys with increased proteinuria and AKI.

Throughout the pandemic, analyses of federal, state, and local data continue to indicate that Black and Hispanic patients suffered a disproportionate burden of COVID-19 cases and deaths. Large disparities in the number of COVID-19 cases and deaths in Black communities may be related to socioeconomic factors, but genetic predisposition to AKI and renal insufficiency could contribute to this burden. Early identification of at-risk patients could lead to proactive drug therapy lessening the impact of COVID-19, for example through use of monoclonal antibodies or the protease inhibitors currently under investigation.

\section{Public Health Significance}

The ability to diagnose and monitor renal disease progression in the context of comorbidities, such as obesity, diabetes, hypertension, and infectious diseases, has become a public health focus that will benefit from NGS testing strategies. Future considerations, including testing availability for vulnerable populations, is critical in order to monitor individuals with renal predisposition and a high-risk genotype.

Since 2020, numerous studies reported that patients with two APOL1 risk alleles have an increased likelihood for collapsing glomerulopathy, with COVID-19 representing the "second hit." Shetty et al reported that a single $A P O L 1$ risk allele may be associated with an increased risk of COVID-19 associated nephropathy. ${ }^{12}$

Knowing that $13 \%$ of Black Americans are at high risk of kidney disease due to the APOL1 risk alleles, how do we ensure that underserved communities at the highest risk of CKD are prioritized for COVID-19 testing and treatment? If a healthcare provider is unaware that an individual is positive for risk alleles, they would be unprepared for rapid progression to end stage kidney disease. ${ }^{11}$ Implications for the Black communities would be to identify those individuals who would most benefit from COVID-19 vaccination and early treatment opportunities. Consequently, long term outcomes are unknown regarding COVID-19 collapsing glomerulopathy.

\section{Acknowledgements}

We wish to acknowledge support from Erin and Christopher Lee and the Piper's Kidney Bean Foundation for the development of the NGS gene panel in the MDL and Dr. Joshua Zaritsky for his insightful discussions during the development of the SRNS panel. We thank Morgan 
Doi: $10.32481 /$ djph.2021.12.008

Thomas, genetic counselor with the Molecular Diagnostics Laboratory, for assistance with gene variant analysis.

These authors can be contacted at susan.kirwin@,nemours.org

\section{References}

1. Whyte, D. A., \& Fine, R. N. (2008, October). Chronic kidney disease in children. Pediatrics in Review, 29(10), 335-341. PubMed https://doi.org/10.1542/pir.29.10.335

2. Harshman, L. A., \& Zepeda-Orozco, D. (2016, March). Genetic considerations in pediatric chronic kidney disease. Journal of Pediatric Genetics, 5(1), 43-50. PubMed https://doi.org/10.1055/s-0035-1557111

3. Amlie-Wolf, L., Baker, L., Hiddemen, O., Thomas, M., Burke, C., Gluck, C., ... Gripp, K. W. (2021, April). Novel genetic testing model: A collaboration between genetic counselors and nephrology. American Journal of Medical Genetics. Part A, 185(4), 1142-1150. PubMed https://doi.org/10.1002/ajmg.a.62088

4. Nicholas Cossey, L., Larsen, C. P., \& Liapis, H. (2017, August). Collapsing glomerulopathy: A 30-year perspective and single, large center experience. Clinical Kidney Journal, 10(4), 443-449. PubMed https://doi.org/10.1093/ckj/sfx029

5. Genovese, G., Friedman, D. J., Ross, M. D., Lecordier, L., Uzureau, P., Freedman, B. I., . . . Pollak, M. R. (2010, August 13). Association of trypanolytic ApoL1 variants with kidney disease in African Americans. Science, 329(5993), 841-845. PubMed https://doi.org/10.1126/science.1193032

6. Yusuf, A. A., Govender, M. A., Brandenburg, J.-T., \& Winkler, C. A. (2021, April 26). Kidney disease and APOL1. Human Molecular Genetics, 30(R1), R129-R137. PubMed https://doi.org/10.1093/hmg/ddab024

7. Freedman, B. I., Divers, J., \& Palmer, N. D. (2013, December). Population ancestry and genetic risk for diabetes and kidney, cardiovascular, and bone disease: Modifiable environmental factors may produce the cures. Am J Kidney Dis, 62(6), 1165-1175. PubMed https://doi.org/10.1053/j.ajkd.2013.05.024

8. Velez, J. C. Q., Caza, T., \& Larsen, C. P. (2020, October). COVAN is the new HIVAN: The re-emergence of collapsing glomerulopathy with COVID-19. Nature Reviews. Nephrology, 16(10), 565-567. PubMed https://doi.org/10.1038/s41581-020-0332-3

9. May, R. M., Cassol, C., Hannoudi, A., Larsen, C. P., Lerma, E. V., Haun, R. S., . . Caza, T. N. (2021, August 3). A multi-center retrospective cohort study defines the spectrum of kidney pathology in Coronavirus 2019 Disease (COVID-19). Kidney International, 13031315. PubMed

10. Sadowski, C. E., Lovric, S., Ashraf, S., Pabst, W. L., Gee, H. Y., Kohl, S., .. .. Hildebrandt, F., \& the SRNS Study Group. (2015, June). A single-gene cause in $29.5 \%$ of cases of steroid-resistant nephrotic syndrome. J Am Soc Nephrol, 26(6), 1279-1289. PubMed

11. Freedman, B. I., Burke, W., Divers, J., Eberhard, L., Gadegbeku, C. A., Gbadegesin, R., .. . Blacksher, E. (2021, April 14). Diagnosis, education, and care of patients with APOL1- 
associated nephropathy: A Delphi consensus and systematic review. J Am Soc Nephrol, 32(7), 1765-1778. PubMed https://doi.org/10.1681/ASN.2020101399

12. Shetty, A. A., Tawhari, I., Safar-Boueri, L., Seif, N., Alahmadi, A., Gargiulo, R., . . . Quaggin, S. E. (2021, January). COVID-19-associated glomerular disease. J Am Soc Nephrol, 32(1), 33-40. PubMed https://doi.org/10.1681/ASN.2020060804

Copyright (c) 2021 Delaware Academy of Medicine / Delaware Public Health Association.

This is an Open Access article distributed under the terms of the Creative Commons Attribution Non-Commercial License (https://creativecommons.org/licenses/by-nc-nd/4.0/) which permits unrestricted non-commercial use, distribution, and reproduction in any medium, provided the original work is properly cited. 